\title{
A view of the Galactic halo using beryllium as a time scale
}

\author{
Rodolfo Smiljanic $^{1} \dagger$, L. Pasquini ${ }^{2}$, P. Bonifacio ${ }^{3,4,5}$, D. Galli ${ }^{6}$, \\ B. Barbuy ${ }^{1}$, R. Gratton ${ }^{7}$, and S. Randich \\ ${ }^{1}$ IAG, Universidade de São Paulo, São Paulo, Brazil \\ email: rsmiljan@eso.org
}

\begin{abstract}
${ }^{2}$ ESO, Garching bei München, Germany, ${ }^{3}$ GEPI Observatoire de Paris - Meudon, France, ${ }^{4}$ INAF, Osservatorio di Trieste, Trieste, Italy, ${ }^{5}$ CIFIST Marie Curie Excellence Team, ${ }^{6}$ INAFOsservatorio di Arcetri, Firenze, Italy ${ }^{7}$ INAF-Osservatorio di Padova, Padova, Italy,
\end{abstract}

\begin{abstract}
Beryllium stellar abundances were suggested to be a good tracer of time in the early Galaxy. In an investigation of its use as a cosmochronometer, using a large sample of local halo and thick-disk dwarfs, evidence was found that in a $\log (\mathrm{Be} / \mathrm{H})$ vs. $[\alpha / \mathrm{Fe}]$ diagram the halo stars separate into two components. One is consistent with predictions of evolutionary models while the other is chemically indistinguishable from the thick-disk stars. This is interpreted as a difference in the star formation history of the two components and suggests that the local halo is not a single uniform population where a clear age-metallicity relation can be defined.
\end{abstract}

Keywords. stars: abundances - stars: late-type - Galaxy: halo

\section{Introduction}

Be is a pure product of cosmic-ray spallation in the ISM involving mostly CNO nuclei. Abundances of $\mathrm{Be}$ in metal-poor stars show a linear relation with $\mathrm{Fe}$ (and $\mathrm{O}$ ) with a slope close to one, implying that Be behaves as a primary element (Smiljanic et al. 2009, and references therein). If cosmic rays are globally transported across the Galaxy, the production of Be is a widespread process and Be abundances should have a smaller scatter than the products of stellar nucleosynthesis at a given time in the early Galaxy (Suzuki \& Yoshii 2001). In other words Be should be a good tracer of time.

Pasquini et al. $(2004,2007)$ calculated Be abundances in turn-off stars of two globular clusters, NGC 6397 and NGC 6752. The Be ages derived from a model of the evolution of Be with time (Valle et al. 2002) were shown to agree with those derived from theoretical isochrones, supporting the use of Be as a cosmochronometer.

Pasquini et al. (2005) analyzed a sample of 20 halo and thick-disk stars and found a possible separation between stars of the two components in a $\log (\mathrm{Be} / \mathrm{H})$ vs. $[\alpha / \mathrm{Fe}]$ diagram. This was interpreted as a difference in the time scales of star formation.

Smiljanic et al. (2009) analyzed the largest sample of halo and thick-disk stars to date, extending the investigation of Be as cosmochronometer and its role as a discriminator of different stellar populations in the Galaxy. The thick disk was found to be a homogeneous population. The halo stars were found to divide into two different components (Fig. 1).

\section{The Galactic halo}

In a diagram of $[\mathrm{O} / \mathrm{Fe}]$ vs. $\log (\mathrm{Be} / \mathrm{H})$ the abscissa can be considered as increasing time and the ordinate as the star formation rate. In Smiljanic et al. (2009) oxygen abundances

$\dagger$ Present address: ESO, Garching bei München, Germany. 

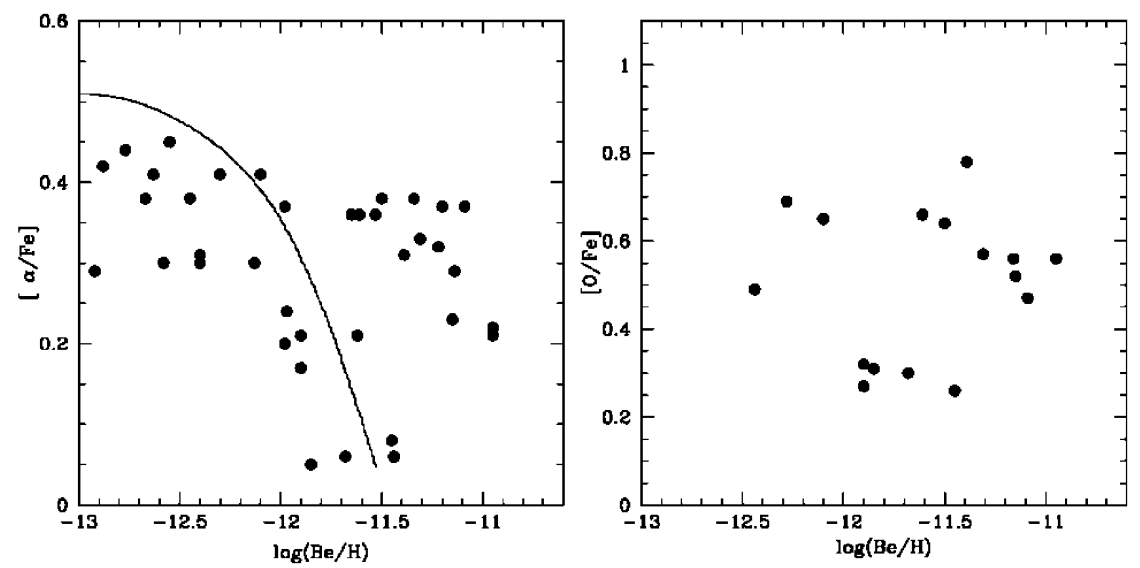

Figure 1. (a) $\log (\mathrm{Be} / \mathrm{H})$ vs. $[\alpha / \mathrm{Fe}]$ for the halo stars of Smiljanic et al. (2009). The solid line is the model prediction of Valle et al. $(2002)$. (b) $\log (\mathrm{Be} / \mathrm{H})$ vs. $[\mathrm{O} / \mathrm{Fe}]$ with new preliminary oxygen abundances for a subsample of the halo stars., corrected for NLTE (Fabbian et al. 2009)

were not available, so mean abundances of $\alpha$-elements were used instead. Here we present new preliminary oxygen abundances determined from the infrared triplet at $777 \mathrm{~nm}$.

As shown in Fig. 1, using either $\alpha$ or oxygen abundances, the halo stars define two clear distinct sequences. One sequence is chemically similar to the thick disk, the other agrees with the models of Valle et al. (2002). The latter, however, with $[\alpha / \mathrm{Fe}] \leqslant 0.25$ and $\log (\mathrm{Be} / \mathrm{H}) \leqslant-11.4$, have similar kinematics. The stars have mostly velocity in the direction of the rotation of the Galaxy $\mathrm{V} \sim 0$ and the perigalactic distance $\mathrm{R}_{\mathrm{min}} \leqslant 1 \mathrm{kpc}$ (see Smiljanic et al. 2009 for details), as expected for accreted stars.

The splitting into two components may be related to the accretion of external systems or to variations of star formation in different and initially independent regions of the early halo. The interpretetion is still open, it is however clear that the halo is not a single uniform population with a single age-metallicity relation. A similar division was found by Nissen \& Schuster $(1997,2009)$ but using Fe as a tracer of time. The division is clearer when Be is used as a time scale. In the same line, recent simulations of the formation of disk galaxies in a $\Lambda$ CDM universe by Zolotov et al. (2009) show that the inner halo has a dual nature, it is composed both by 'in situ stars' formed in the potential well of the galaxy and by accreted stars, formed in subhalos later accreted by the galaxy.

\section{Acknowledgements}

R.S. acknowledges financial support from FAPESP (04/13667-4 and 08/55923-8).

\section{References}

Fabbian, D., Asplund, M., Barklem, P. S., et al. 2009, A\&A, 500, 1221

Nissen, P. E. \& Schuster, W. J. 1997, A\&A, 326, 751

Nissen, P. E. \& Schuster, W. J. 2009, in: Proc. IAU Symposium No. 254, p. 103

Pasquini, L., Bonifacio, P., Randich, S., Galli, D., \& Gratton, R. G. 2004, A\&A, 426, 651

Pasquini, L., Bonifacio, P., Randich, S., et al. 2007, A\&A, 464, 601

Pasquini, L., Galli, D., Gratton, R. G., et al. 2005, A\&A, 436, L57

Smiljanic, R., Pasquini, L., Bonifacio, P., et al. 2009, A\&A, 499, 103

Suzuki, T. K. \& Yoshii, Y. 2001, ApJ, 549, 303

Valle, G., Ferrini, F., Galli, D., \& Shore, S. N. 2002, ApJ, 566, 252

Zolotov, A., Willman, B., Brooks, A. M., et al. 2009, ApJ, 702, 1058 


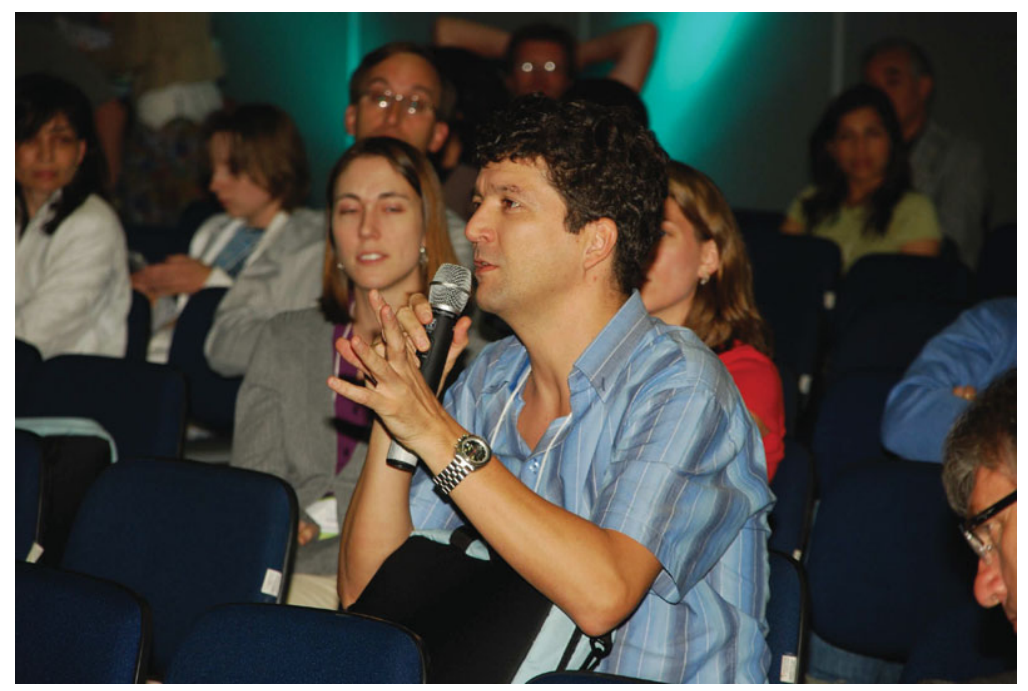

Ricardo Schiavon in the audience.

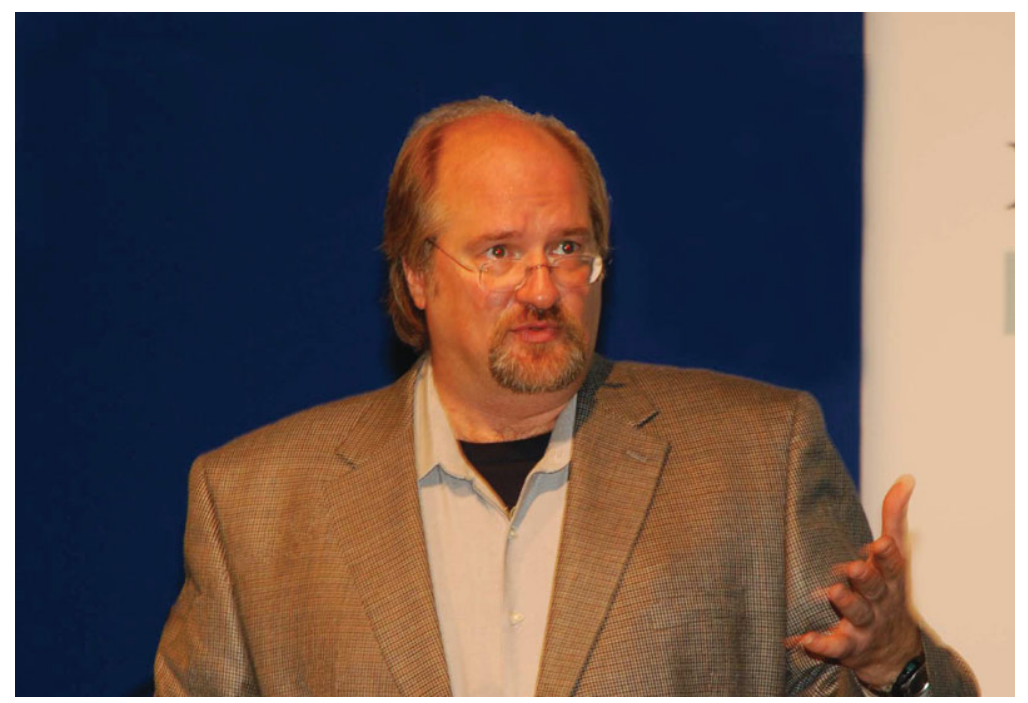

Steve Majewski chairing a session. 